\title{
ROUTING AND COMMUNICATION PATH MAPPING IN VANETS
}

\author{
Nnamdi Anyameluhor and Dr Evtim Peytchev \\ School of Science and Technology \\ Nottingham Trent University \\ Clifton Lane, Nottingham, NG11 8NS, UK \\ E-mail: \{nnamdi.anyameluhor2012, evtim.peytchev\}@ntu.ac.uk
}

\section{KEYWORDS}

Vehicular Ad-Hoc Network; Cross-layer; Routing; Network Simulation; ITS; NS-3; Communication Mapping; MAC; Mobile Programming;

\begin{abstract}
Vehicular ad-hoc network (VANET) has quickly become an important aspect of the intelligent transport system (ITS), which is a combination of information technology, and transport works to improve efficiency and safety through data gathering and dissemination. However, transmitting data over an ad-hoc network comes with several issues such as broadcast storms, hidden terminal problems and unreliability; these greatly reduce the efficiency of the network and hence the purpose for which it was developed. We therefore propose a system of utilising information gathered externally from the node or through the various layers of the network into the access layer of the ETSI communication stack for routing to improve the overall efficiency of data delivery, reduce hidden terminals and increase reliability. We divide route into segments and design a set of metric system to select a controlling node as well as procedure for data transfer. Furthermore we propose a system for faster data delivery based on priority of data and density of nodes from route information while developing a map to show the communication situation of an area. These metrics and algorithms will be simulated in further research using the NS-3 environment to demonstrate the effectiveness.
\end{abstract}

\section{INTRODUCTION}

When the Fleetneet project in Germany promised better route information, traffic and road condition updates and socialising on the road in 2001 (Hartenstein et al. 2001) it seemed a bit futuristic but nowadays we see that these kinds of vehicular services should be a necessity in order to increase comfort and safety of road users as well as greatly improve traffic management systems. This 'lofty' idea is part of the Intelligent Transport System that has being developed and is continually evolving as a lot of research and interest is shown in the area. The Intelligent Transport System (ITS) is simply the combination of telecommunication systems, information technology and transport system to provide relevant information to road users for the sake of safety and efficiency (Bishop 2005).

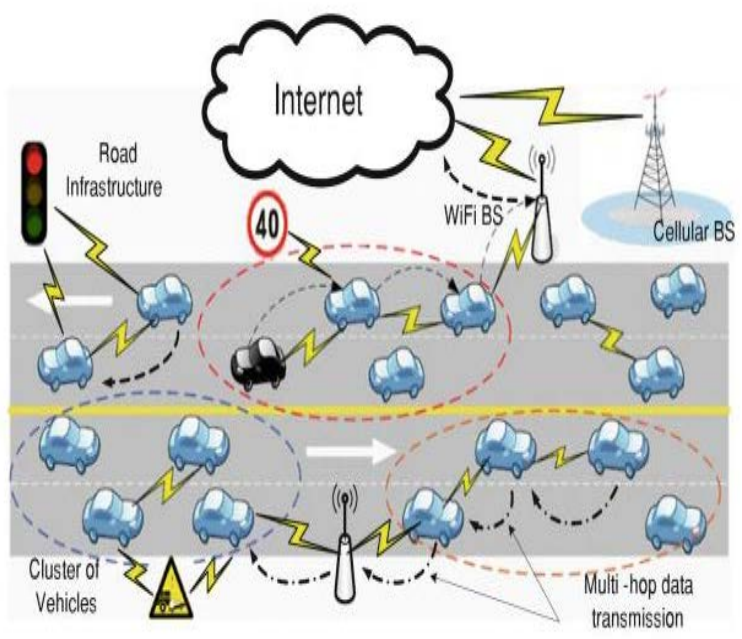

Figure 1: VANET Concept (Zhu and Li 2013)

In order to disseminate information two methods can be used which are cellular networks and other wireless short-range definitions such as those defined by the Cooperative Intelligent Transport Systems (C-ITS). Whereas cellular networks work through a centralised topology with standard infrastructure (Base Station etc.) regardless of the position of the transmitting and receiving nodes, C-ITS on the other hand defines a framework where direct wireless networked devices can form an ad-hoc networks. When vehicles form ad-hoc networks by connecting to each other within range it is called a vehicular ad-hoc network (VANET), it is a highly mobile network where links can be created and broken in seconds (Karagiannis et al. 2011) therefore it is of high importance to have a system that can cope with this mobility while being able to maintain connection and data flow. In 2009 the European commission issued a formal request (Mandate 453) to CEN and ETSI, to prepare a set of standards and specifications to support the implementation of Cooperative ITS systems across Europe (ETSI 2014) The ETSI have developed an adapted OSI network framework to accommodate ITS applications and design as seen in figure 2 


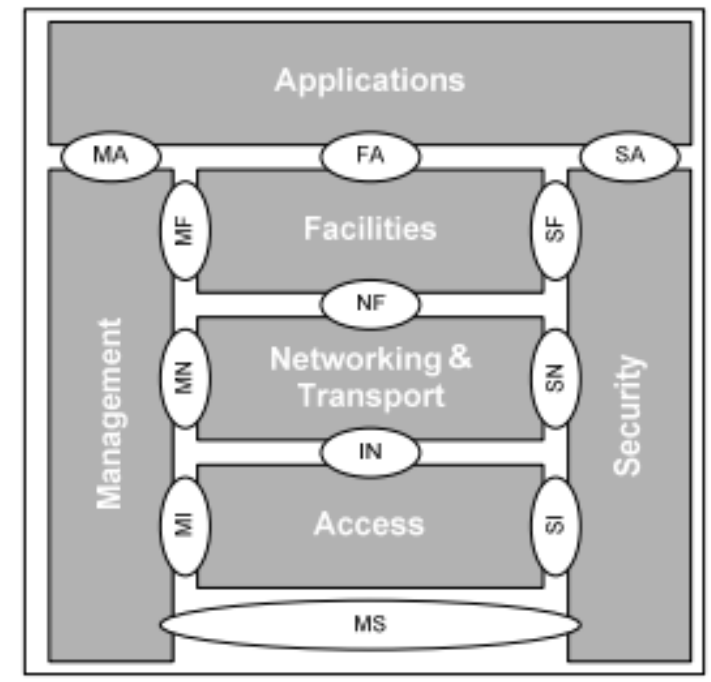

Figure 2: ITS Architecture

Data loss, packet collision, broadcast storms and hidden terminal problems are some major issues that may be faced in vehicular ad hoc networks because the nature of the wireless medium as well as change in network topology due to the obvious reasons of mobility and speed (Yousefi et al. 2006). Pure flooding broadcast and other traditional broadcast schemes (without acknowledgment) may not be efficient enough to ensure reliable data spread (Karagiannis et al. 2011). Also the communication knowledge of an area in VANETs has been neglected in research, this knowledge is very important in terms of message dissemination and other decision-making processes.

In this paper we propose a system for utilisation of cross layer information from the physical layer and network layer to improve cluster formation and for relaying of data; this also includes data priority while service messages are in a contention loop as well as channel selection process. We also propose the use of node density algorithms to construct a communication map of the area.

\section{RELATED WORK}

Efficiency and reliability are important factors in the design of VANET protocols, especially in cases of emergencies. Unfortunately these factors are not adequately catered for in the default architecture due to the use of a MAC protocol based on IEEE 1609.4 and IEEE 802.11p (Sjöberg 2013). This protocol employs the use of CSMA in the MAC layer which has been shown to perform poorly as the number of nodes increase in the network due to an equally increased probability of two or more nodes having the same backoff counter for attempted packet delivery (Bilstrup et al. 2008), the result of which is packet collisions.

This disadvantage has led to a lot of research focused on improving the throughput' of packets in the network.
Among these research Zhou et al. (2008) proposed a cross layer cooperative VANET with rate and medium access control, they introduced cooperative communication and cross layer link detection at the MAC, network and transport level in order to improve message dissemination and fairness of medium access. However, this system employs the use of roadside units or "gateways" to achieve this aim, this will no doubt add to the overall cost of the VANET architecture and reliance on extra hardware should as much as possible be minimised. The AMB protocol (Korkmaz 2009) requires a node to propagate a "black burst" or jamming signal as far as possible within the network with the furthest node responding and hence becoming the next relay for messages. The drawback of this system is the time delay involved in the sending and response of this black burst. In research done by Bi et al. (2009) nodes have reduced time delay but the work focuses message propagation based on direction. De Couto et al. (2005) proposed metric for high throughput in multi-hop networks by taking into account the probability ratio of expected transmission count in effective message delivery to the number of attempted transmission. Cross layer broadcast protocol by Bi et al. (2010) describes a system for efficient routing and network organisation in order to tackle the hidden terminal problem and the broadcast storm issue by using a request to send and clear to send (RTS/CTS) scheme. This RTS/CTS is their response to the lack of acknowledgement in the CSMA protocol. Our protocol combines cross layer functionality to provide efficient and reliable routing; cluster aggregation of nodes to improve fairness, avoid broadcast storms and hidden terminals; TDMA and transmission request priority to avoid collision and finally a system to generate communication maps of an area.

\section{PROPOSED WORK}

\section{Cluster head Selection}

Hafeez et al. (2011) proposed a cluster and OFDMA MAC protocol with each cluster assigned a set of subcarrier to distinguish it from other clusters, cluster heads are established based mainly on a weighted stability factor on the road. While the system increases reliability and reduces delay there is the issue of the system adapting or performing self-learning processes to maintain this reliability. Also due to wireless ranges the choice of making each cluster the size of two times the range of the cluster head's transmission may be inhibiting as the cluster head may struggle to reach every member of the cluster.

For this research we also intend to create a cluster of nodes by dividing the network into small groups with a viable cluster head whose primary duty includes maintaining stability of the network and data flow. By creating small clusters we hope to form and maintain a 
network within a short period while enabling a more robust system for data dissemination along the route.

In Figure 2 it can be noted that there is a Facility layer in the ETSI model and among the duties of the Facilities layer include; support of getting and combining data, channel selection etc. Therefore the following description can be processed and shared within the facility layers of nodes in a one hop radius with respect to selecting a cluster head.

Since the ETSI standard covers an application sensitive layer, we propose an internal control worthiness metric to calculate which node should be the cluster head within each cluster. The data used in this calculation will include Nodal Age on Route (NAR), Position Relative to other Nodes (PRON), Direction (DIR), Speed and Acceleration (SACC) and Node Equipment Status (NES); we assume the presence of these information and will set them dynamically for this research. Algorithms and processes of how to gather data have been done in research carried out by Gamati (2012). Together the information is used to develop a Master Probability Metric (MPM) system by assigning values and corresponding Impact Factors (IMF) to the information and sorting them according to the nodes with the highest impact, the highest then selected as the cluster head.

Table 1: Information, Value and Impact Factor for Determining Suitable Cluster Head.

\begin{tabular}{|c|c|c|}
\hline INFORMATION & VALUE & $\begin{array}{l}\text { IMPACT } \\
\text { FACTOR }\end{array}$ \\
\hline \multirow[t]{2}{*}{ Position } & Central & 9 \\
\hline & Corner & 1 \\
\hline \multirow[t]{2}{*}{ Direction } & Corresponding & 9 \\
\hline & Opposite & 1 \\
\hline \multirow{2}{*}{$\begin{array}{l}\text { Speed and } \\
\text { acceleration } \\
\text { relative to other } \\
\text { nodes }\end{array}$} & High & 1 \\
\hline & Steady & 9 \\
\hline \multirow{2}{*}{$\begin{array}{l}\text { Age on route } \\
\text { (communication } \\
\text { map metric) }\end{array}$} & Lengthy & 9 \\
\hline & Short & 1 \\
\hline
\end{tabular}

From Table 1 it can be noted that a central node (relative to other nodes) with a steady speed and low acceleration has a better chance of being elected a cluster head. In order to calculate these process we will consider the selection as a multi-dimensional knapsack issue where the aim is to maximise a choice outcome based on several constraints:
Maximise

$$
\sum_{j=1}^{n} P j X j
$$

Subject to:

$\sum_{j=1}^{n} W i j X j \leq W i$, for all $1 \leq i \leq m$

$$
X j \geq 0, X j \text { integer for all } 1 \leq j \leq n
$$

The algorithm takes the summation of the values and impact factors and must pick a solution while satisfying the stated constraints. Given that this computation may take up to a few seconds to complete, this system may be insufficient for emergency purposes and better suited to nodes in a platoon formation.

\section{Data Routing}

According to the ETSI standard for C-ITS message priority may be handled in two ways which are; transmission request from the Data Link Layer and those from the PHY layer both in the Access block. The transmission request from the DLL handles messages from the 'Application' block of the station while the request from the PHY layer handles contention of different ITS stations in the physical communication channel (ETSI 2013). This second transmission contention between different nodes is what we look into in this proposal.

At the end of the cluster head selection process and network stabilization, data may now be routed among members of the cluster and surrounding with TDMA through the cluster head using the CALM (Communication Access for Land Mobiles) M5 protocol. We propose the use of TDMA in place of CDMA in order to avoid unnecessary packet collisions in the system. In this process every message is given a class and priority value e.g. 1 for emergency, 2 for critical messages, 3 for network initialisation etc. this is done for the sole purpose of facilitating routing at the MAC level and making sure priority messages are given due resources.

Table 2 Example of Data Type and Priority Value

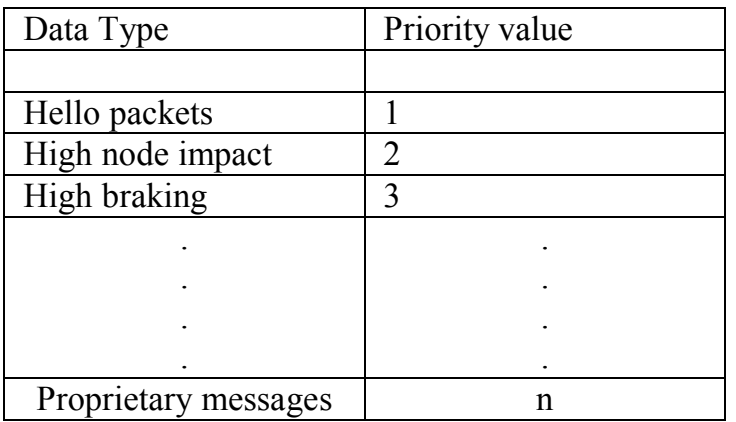


The messages are polled by the cluster head and shared in the cluster according to the process described by the flow chart in Figure 3.

The highest priority message is given the next available time slot and resource while other data is reorganised accordingly with the exit or addition of packets.

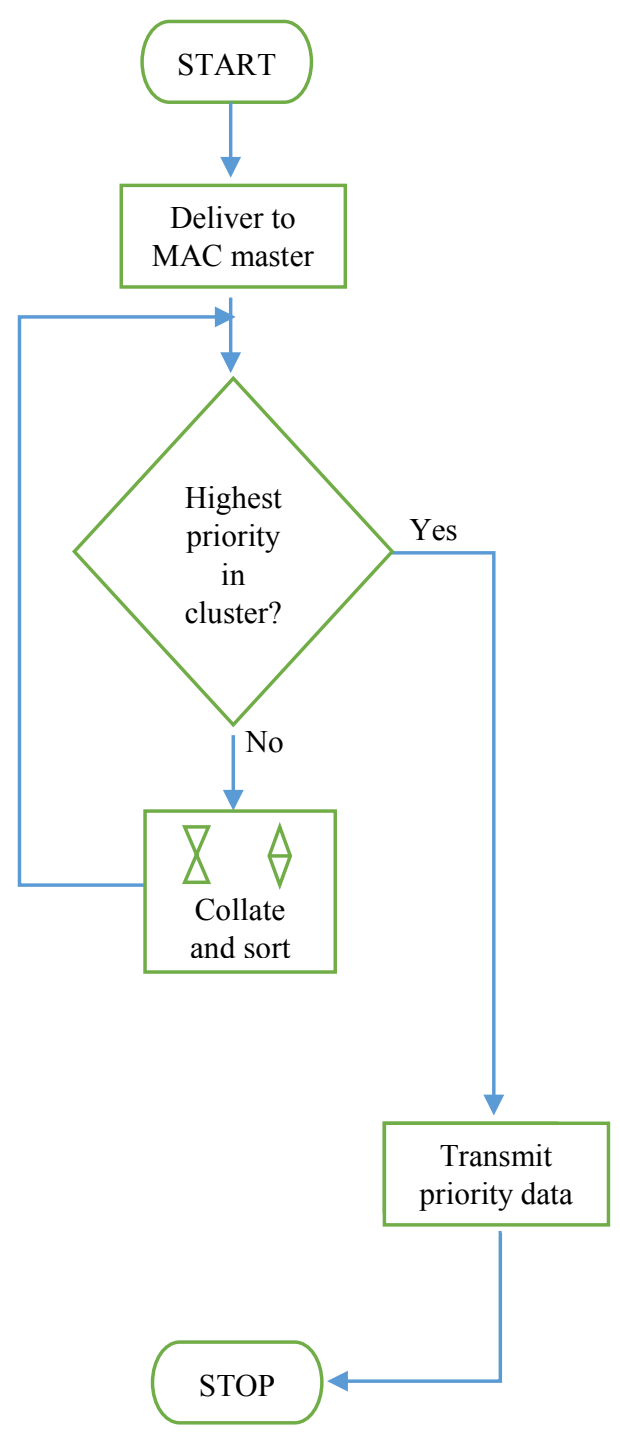

Figure 3: Flow chart for data transmission

\section{COMMUNICATION MAPPING}

Mapping has always been a simple way of communication information to an end user and the focus of work so far has been on providing useful information and traffic condition information, however we believe that the nodes can provide other useful information to aid building of networks across larger areas, hence in this case we propose a system to draw up not routing information but communication information for a section of a city based on both prior and current information example seen in the Figure 4.

By using node density algorithms and past node history we aim to be able to draw a map to describe how the communication network in a certain area is at a particular time e.g. Assuming each coloured path represents a route within a city, this paths have had their communication situation analysed with respect to node density, gathered node information, channel conditions etc. we represent the conditions as particular colours in this case red represents very poor communication conditions and green represents good communication condition.

This we hope will help reduce the decision time for routing and network management

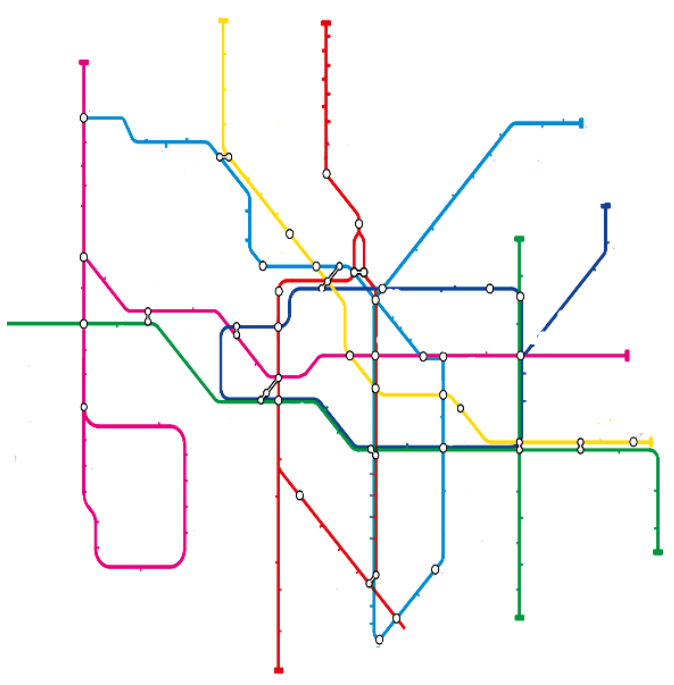

Figure 4: Communication map model with different colours indicating different network conditions

\section{CONCLUSION}

In this paper we have proposed a system to create and maintain network and routing stability for nodes in a platoon arrangement by utilising data from the physical world; it involves selecting a Mac controller or cluster head and forming node segments. We also proposed the use of TDMA for the control and service channels in VANETs in place of the original CDMA due packet collisions and reliability. Finally we have also proposed a communication mapping system based on node density algorithms. Our future work will be to simulate these proposals using NS3 and measure the performance against both the IEEE $802.11 \mathrm{p}$ standard and other popular suggestions. The concept of VANET is one that aims to reduce accidents, aid better traffic management and on-road sales and services, therefore will benefit from a more stable design as proposed. 


\section{REFERENCES}

Agarwal, A., Starobinski, D. and Little, T.D.C. (2007) 'Exploiting downstream mobility to achieve fast upstream message propagation in vehicular ad hoc networks', in 2007 Mobile Networking for Vehicular Environments, May, 11 November. Anchorage, AK, pp.13-18.

Bishop, R. (2005) Intelligent Vehicle Technologies and Trends, Artech House, Boston, Mass, USA.

Bi, Y., Cai, L.X., Shen, X. and Zhao, H., 2010. A cross layer broadcast protocol for multihop emergency message dissemination in inter-vehicle communication. In: Communications (ICC), 2010 IEEE International Conference on, IEEE, pp. 1-5.

Bi, Y., Zhao, H. and Shen, X., 2009. A directional broadcast protocol for emergency message exchange in intervehicle communications. In: Communications, 2009. ICC'09. IEEE International Conference on, IEEE, pp. 15.

Bilstrup, K., Uhlemann, E., Strom, E.G. and Bilstrup, U., 2008. Evaluation of the IEEE 802.11 p MAC method for vehicle-to-vehicle communication. In: Vehicular Technology Conference, 2008. VTC 2008-Fall. IEEE 68th, IEEE, pp. 1-5.

De Couto, D.S., Aguayo, D., Bicket, J. and Morris, R., 2005. A high-throughput path metric for multi-hop wireless routing. Wireless Networks, 11 (4), 419-434.

ETSI, 2013. Intelligent Transport Systems (ITS); Communications Architecture Standard [online]. ETSI. Available http://www.etsi.org/deliver/etsi_en/302600 302699/302 665/01.01.01 60/en 302665v010101p.pdf [Accessed $\mathrm{Feb} / 4$ 2014].

ETSI Cooperative ITS 2014. [Online]. ETSI. Available at: http://www.etsi.org/technologiesclusters/technologies/intelligent-transport/cooperativeits [Accessed Feburary/4 2014].

Gamati, E., Peytchev, E. and Germon, R., 2013. Utilization of Broadcast Methods for detection of the road conditions in VANET. Nottingham Trent University - School of Science and Technology - Computing and Informatics.

Hafeez, K.A., Zhao, L., Liao, Z. and Ma, B.N., 2011. Clustering and OFDMA-based MAC protocol (COMAC) for vehicular ad hoc networks. EURASIP Journal on Wireless Communications and Networking, 2011 (1), 1-16.

Hartenstein, H., Bochow, B., Ebner, A., Lott, M., Radimirsch, M. and Vollmer, D., 2001. Position-aware ad hoc wireless networks for inter-vehicle communications: the Fleetnet project. In: Proceedings of the 2nd ACM international symposium on Mobile ad hoc networking \& computing, ACM, pp. 259-262.

Karagiannis, G., Altintas, O., Ekici, E., Heijenk, G., Jarupan, B., Lin, K. and Weil, T., 2011. Vehicular networking: A survey and tutorial on requirements, architectures, challenges, standards and solutions. Communications Surveys \& Tutorials, IEEE, 13 (4), 584-616.

Korkmaz, G., Ekici, E. and Ozguner, F., 2007. Black-burstbased multihop broadcast protocols for vehicular networks. Vehicular Technology, IEEE Transactions on, 56 (5), 3159-3167.

Sjöberg, K., 2013. Medium Access Control for Vehicular Ad Hoc Networks. Doctor of Philosophy, Chalmers University of Technology; Halmstad University.
Taniguchi, E., Thompson, R.G., Yamada, T. and Van Duin, R., 2001. City Logistics. Network modelling and intelligent transport systems.

Yousefi, S., Mousavi, M.S. and Fathy, M., 2006. Vehicular ad hoc networks (VANETs): challenges and perspectives. In: ITS Telecommunications Proceedings, 2006 6th International Conference on, IEEE, pp. 761-766.

Zhou, L., Zheng, B., Geller, B., Wei, A., Xu, S. and Li, Y., 2008. Cross-layer rate control, medium access control and routing design in cooperative VANET. Computer Communications, 31 (12), 2870-2882.

Zhu, H., and Li, M., 2013. Studies on Urban Vehicular Ad-hoc Networks. 1st Ed. New York: Springer New York.

\section{AUTHOR BIOGRAPHIES}

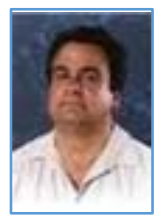

DR. EVTIM PEYTCHEV is Reader in Wireless, Mobile and Pervasive Computing in the school of Science and Technology at Nottingham Trent University, UK. He is leading the Intelligent Simulation, Modelling and Networking Research Group, which consist of 5 lecturers, 3 Research Fellows and 6 research students. He is the Module Leader for Systems Software; and Wireless and Mobile Communications. He also teaches on the modules Software Design and Implementation; Mobile Networking; Enterprise Computing; and Computer Architecture.

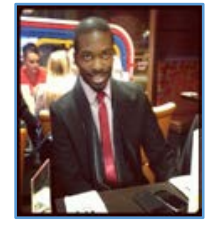

NNAMDI ANYAMELUHOR went to the University of Science and Technology (Nigeria) where he recieved his BTech in Electrical and Electronic Engineering, he then went to study Cybernetics and Communication Engineering at Nottingham Trent University (United Kingdom) where he obtained his MSc. He is currently a Post Graduate researcher in Nottingham Trent University in the field of Vehicular Ad Hoc Networks. 